\title{
Hypothesis
}

\section{Does junk DNA regulate gene expression in humans?}

\author{
M A Hultén, M Stacey, S J Armstrong
}

It has been said that the fruitfly, Drosophila (of which there are many species, the most well known being Drosophila melanogaster), is an organism created for the benefit of geneticists. Many basic genetic principles, conserved and applicable to higher organisms including humans, have been discovered initially by the study of fruitflies (which have a short generation time of around two weeks, and where colonies may be easily maintained in bottles of nutrient agar). Thus, for example, Muller discovered in the early 1930s that exposure of Drosophila to ionising radiation induces both single gene mutations and structural chromosome rearrangements. ${ }^{1}$ During the course of his investigations, Muller also found that some such chromosome rearrangements, when placing euchromatic genes adjacent to heterochromatin (now often called junk DNA), led to changes in the expression of the genes concerned. ${ }^{2}$ The effect on gene expression typically varies between individual cells, leading to a mottled phenotype of, for example, eye colour, which can be identified directly or by the use of a low magnification microscope. The occurrence of a mottled phenotype has led to the phenomenon being called heterochromatin position effect variegation $(\mathrm{PEV}){ }^{34}$

Heterochromatin; which stains differently from euchromatin by common chromosome dyes, is generally accepted as being devoid of structural genes. ${ }^{5}$ This type of chromatin consists predominantly of stretches of repeated DNA sequences, replicates out of phase with euchromatin, and has no known function in higher organisms. In this paper we provide examples from the human situation, indicating that this so called junk DNA may indeed serve an important function, that is in the regulation of tissue specific gene expression. We present a model which is similar to the heterochromatin position effect variegation in Drosophila, where we suggest that the differential packing status of repeated DNA sequences may provide a mechanism for overall regulation of tissue specific gene activation in humans. Therefore, repetitive DNA sequences may now have to be looked upon as "treasured items" rather than "junk".

\section{Heterochromatic regions and repeated DNA sequences}

The term heterochromatin, based on the different chromosome morphology, was first introduced into the genetic vocabulary by Heitz in 1928. He defined heterochromatic regions of chromosomes as those that retain a compact structure during interphase, display a differential condensation at prophase, and unlike euchromatin, do not unravel at telophase of the mitotic cycle. ${ }^{6}$ As already mentioned, these regions differ from euchromatin in being composed predominantly of non-coding, largely repetitive, DNA. They are thus permanently non-transcribable rather than simply repressed, and so represent a distinct structural kind of chromatin in comparison to euchromatin. ${ }^{5}$

It is now known that the human genome contains many different types of repetitive DNA. Some DNA sequences are repeated many thousands of times, and may be identified as heterochromatic blocks under the microscope. Others are only moderately repeated and therefore too small to be resolvable by conventional microscopy. Examples of heterochromatic blocks include the centromeres, the major paracentromeric blocks, and the telomeres. The centromeric regions are made up mainly of "alphoid" repeats of 171 DNA base pairs. ${ }^{7}$ The major paracentromeric heterochromatic blocks (situated adjacent to the centromeres of chromosomes 1,9 , and 16 , as well as occupying the long arm of the $Y$ ) consist of the "classical" satellites I, II, and III, and the telomeres of TT AGGG repeats, about 10000-15000 base pairs long. ${ }^{8}$ The centromeric and in particular the paracentromeric heterochromatic blocks show considerable size variation between individuals, a heteromorphism which does not seem to confer any phenotypic distinction. ${ }^{29}$ This normal heteromorphism has been demonstrated by microscopy following Giemsa staining after partial denaturation by alkali pretreatment, so called C banding, or by Distamycin-DAPI. ${ }^{29}$ More recently specific probes have become available for the selective identification of both centromeres, paracentromeric heterochromatic blocks (see fig 2), and telomeres by in situ hybridisation. ${ }^{10}$

Some types of repetitive DNA have now been intensively studied by molecular technology. ${ }^{11}$ These include tandemly repeated minisatellites, which show a selective positioning within chromosomes. They are preferentially subtelomerically localised. Others, such as 


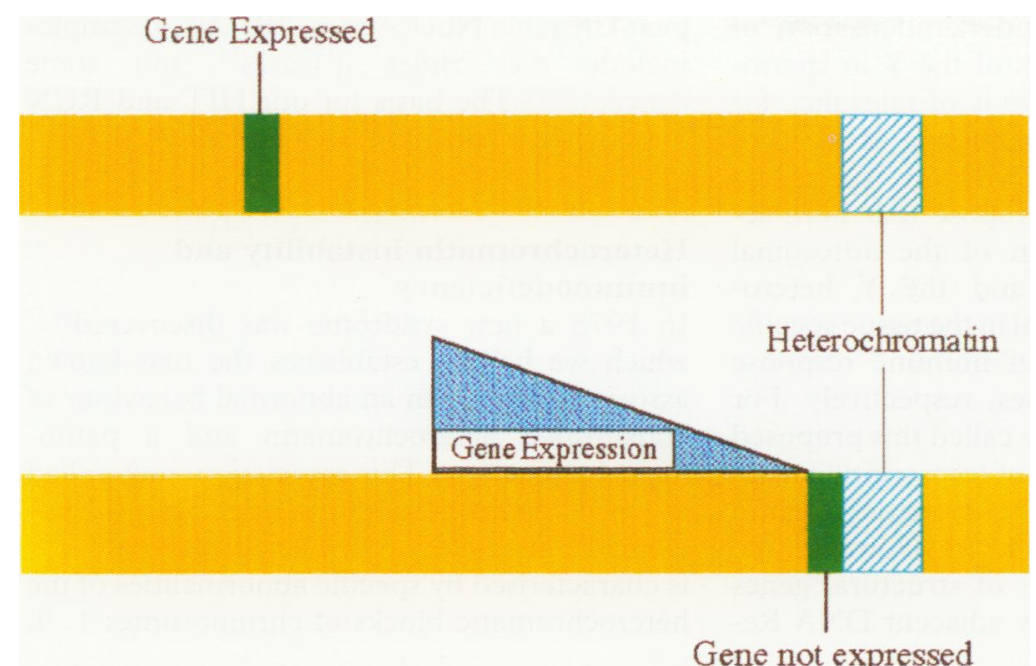

Figure 1 In Drosophila the activity of structural genes is influenced by their positioning in relation to heterochromatic blocks.

tandemly repeated micro-satellites and LINES and SINES, are apparently randomly interspersed along the length of the chromosome. Mini-satellites are about $1000-2000$ base pairs long, and show locus specific repeats with much interindividual variation. For this reason they have been extensively used in forensic science. ${ }^{12}$ Micro-satellites, consisting of di-, tri-, and tetranucleotide tandem repeats, are normally less than 100-200 base pairs long. The trinucleotide repeats have attached much attention recently due to their amplification in so called triplet diseases. ${ }^{13}$

\section{DNA packaging}

The DNA helix of a human cell is estimated to be composed of around 6000 million base pairs. If stretched out, this DNA would measure of the order of one metre. In the living cell, on the other hand, the nuclear DNA is $10000-$ fold packaged into the coiled-folded-coiled chromosome structures. ${ }^{14}$ Firstly the DNA double helix is wrapped twice around eight histones to form the nucleosomal beads, and secondly this $10 \mathrm{~nm}$ beaded structure, composed of nucleosomes and spacer DNA, is coiled by aid of histone $\mathrm{H} 1$-like proteins to form the $30 \mathrm{~nm}$ chromatin fibre. This is yet again folded or coiled into a 200-300 nm thread, which is finally differentially macrocoiled along the length of the chromosome. It is the precise differential macrocoiling that allows identification of individual chromosomes and chromosome segments of the quinacrine or Giemsa banded human chromosome at mitotic prometaphase and metaphase, ${ }^{1516}$ as well as meiotic pachytene prophase ${ }^{17}$ and first and second meiotic metaphase stages. $^{18}$

The exact mechanism behind the DNA chromatin/chromosome packaging still remains an enigma, and may obviously show variation between tissues within organisms and differences between species. It is, however, becoming increasingly accepted that heterochromatin, which in itself shows a selective condensation pattern out of phase with the euchromatin, may be involved in the overall regulation of DNA packaging to include an influence over adjacent euchromatin. It is of further paramount interest that the chromatin condensation per se is thought to be intimately associated with gene expression. ${ }^{19}$ In principle decondensed chromatin is believed to be associated with transcriptional activation of structural genes, while on the other hand condensed chromatin is the rule with gene inactivity. In this sense it follows that the repetitive DNA and its associated proteins could indeed play a most important functional role as a local regulator of gene expression.

\section{The models}

In Drosophila the degree of shutting down of gene activity following displacement near to heterochromatic segments is dependent on the distance between these euchromatic genes and the heterochromatic border (fig 1). The phenotypic effect, the position effect variegation $(\mathrm{PEV}),{ }^{3}$ is normally a recessive character, as may be expected when gene activity of one of the two alleles may phenotypically compensate for a defect of the other. Dominant PEV does, however, also occur, ${ }^{20}$ in which case the defective gene alleles on both chromosomes may be lethal to the carrier individual. It is thought that the expression of all genes in Drosophila may be under the influence of PEV. This system is highly versatile by aid of a large number of modifier genes, which may either, and commonly, enhance the transcriptional silencing, or, more rarely, decrease it. These modifiers are located on other chromosomes, and encode proteins involved in the heterochromatin packaging. ${ }^{21}$

The mechanisms for PEV are not known in any detail. It is, however, accepted that the original idea, based on cytological observations by Prokofieva-Belgovskaya in $1941,{ }^{22}$ is correct, that is, that the dense condensation of heterochromatin may spread into adjacent euchromatin, the transcriptional silencing of genes in these latter segments thus being a direct result of their higher degree of chromatin compaction. ${ }^{3}$ This mechanism would readily explain the more common recessive type of PEV. However, the explanation for dominant PEV must be more complex, as it involves not only a cis effect on the chromatin compaction of the same homologue of a chromosome pair, but also a trans influence on the other homologue. ${ }^{20}$ It has been suggested that this trans information is effected by a somatic pairing mechanism, allowing heterodimerisation of heterochromatin associated proteins involved in the silencing of gene expression. ${ }^{23}$

Our own model is based on cytogenetic observations of heterochromatin instability in two different types of situation in humans. Firstly, the so called ICF syndrome (Immunodeficiency, Centromeric instability, and Facial dysmorphism) comprises a clear indication of a direct relation between a pathological behaviour of heterochromatin and a distinct phenotype. Secondly, we have observed 
a selective differential undercondensation of the heterochromatic block of the $\mathrm{Y}$ in spermatogonia, which we believe is of relevance for the uncoupling of gene expression of adjacent euchromatic genes involved in spermatogenesis. In essence, we propose that both selective undercondensation of the autosomal heterochromatic blocks and the $\mathrm{Y}$ heterochromatin are instrumental in the tissue specific switch on/off processes of immune response and spermatogenesis genes, respectively. For historical reasons, we have called this proposed mechanism for regulation of transcriptional activity "HIT" (an acronym for Heterochromatin Instability in Tissue specificity). However, the differential packing status of structural genes may also be influenced by adjacent DNA Re- peat Unstable Nucleotides, "RUN". Examples include the triplet diseases ${ }^{13}$ and some cancers. ${ }^{24}{ }^{25}$ The basis for our HIT and RUN hypothesis is further described below.

\section{Heterochromatin instability and immunodeficiency}

In 1978 a new syndrome was discovered ${ }^{2627}$ which we believe establishes the first known association between an abnormal behaviour of constitutive heterochromatin and a pathological phenotype. This association, now called the ICF syndrome (Immunodeficiency, Centromeric instability, and Facial dysmorphism) is characterised by specific abnormalities of the heterochromatic blocks of chromosomes 1, 9,
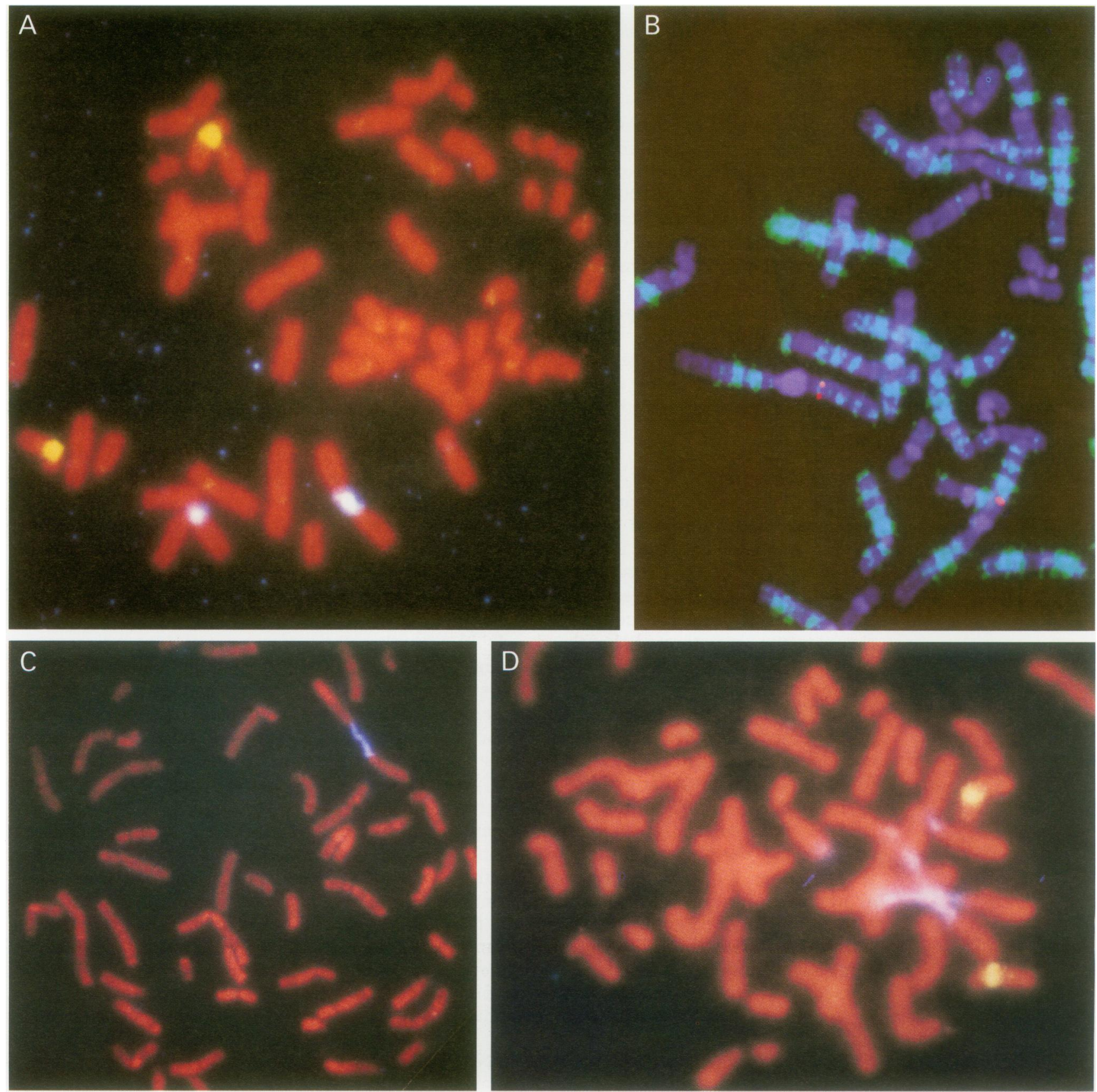

Figure 2 Heterochromatic blocks of chromosome 1 (blue) and chromosome 9 (yellow) in metaphases from human blood lymphocytes. $(A, B)$ Normal metaphases, showing the positioning of the zeta gene (red) adjacent to the heterochromatic block of chromosome 1 in (B). (C,D) Metaphases from ICF patient, illustrating undercondensation and somatic pairing of the heterochromatic blocks of chromosome 1. The probes used for in situ hybridisation were 1 1 h (Cytocell Ltd) specific for the heterochromatic block of chromosome 1; pMR9A specific for the heterochromatic block of chromosome 9 (donated by M. Rocchi, Institute of Genetics, University Degli Studi, Italy), the zeta gene probe (Genome Systems Inc., USA), the whole chromosome X paint (Cambio Ltd), and the X centromere (Cytocell Ltd). 
and 16 , as illustrated in fig $1 \mathrm{C}, \mathrm{D}$. The syndrome is rare, and to date only 14 cases have been published. ${ }^{28}$ Impairment of immune response is the only obligate clinical feature of these patients. The primary ascertainment of cases so far published has been for cytogenetic diagnosis, because of some facial dysmorphism, often in combination with other physical deformities and commonly with psychomotor developmental delay. There is concern that ascertainment bias may have distorted the clin-
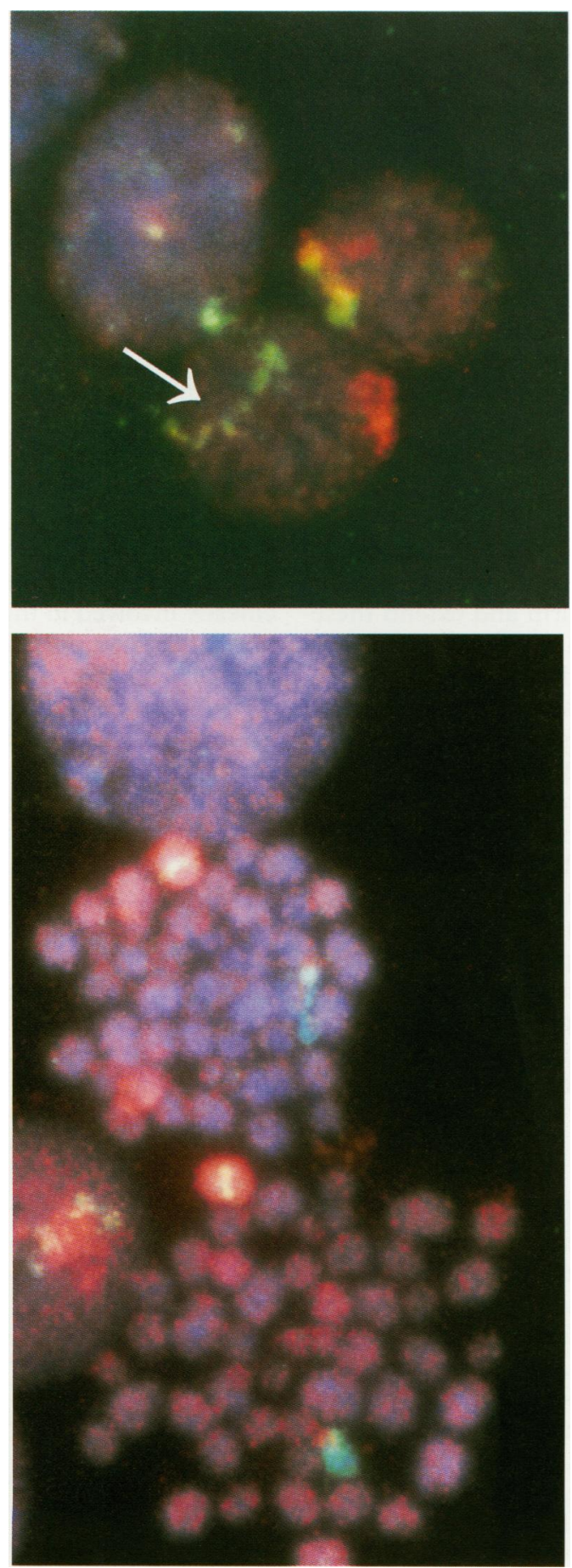

Figure 3 Undercondensation of the heterochromatic block of the $Y$ (green) in the germ line. Top: Interphase gonocyte nucleus from human fetal testis; bottom: spermatogonial metaphases from adult testis. Note undercondensation of the heterochromatic block of $Y$ in the top panel, indicated by arrow. Note also undercondensation in upper cell, but condensation in lower cell in the bottom panel. The whole $X$ is illustrated in red with the $X$ centromere in yellow. ical delineation of this syndrome. No systematic ICF chromosome screening has so far been performed in patients diagnosed as having common variable immunodeficiency. This would be of special interest, as there are brothers and sisters of ICF patients who have no physical malformations and only mild immunodeficiency, but yet show some typical heterochromatin abnormalities. Parents are usually asymptomatic, and it has been concluded that this is a recessive syndrome.

One most interesting feature of the ICF syndrome is that the heterochromatin instability is predominantly expressed in $\mathrm{T}$ lymphocytes. ${ }^{262829}$ This instability primarily concerns an undercondensation and fragility of the heterochromatic blocks of chromosomes 1 , 9, and 16 (fig 2C). These heterochromatic blocks are also seen to be abnormally paired, with apparent recombination between them (fig 2D). All patients show the same typical heterochromatin instability but there is variation with respect to the involvement of the different chromosomes and the proportion of lymphocytes affected.

One further special feature of the chromosome instability of this syndrome concerns the spreading of the undercondensation from the heterochromatic blocks distally into the adjacent euchromatin (Hultén MA, unpublished observations). The degree of this euchromatin undercondensation, cytologically detectable in a small proportion of cells only, may vary substantially between cells; and sometimes pulverisation of all chromosomes may be seen. We propose that this spreading effect may be of crucial importance for the clinical phenotype of the syndrome. Thus we suggest that the primary heterochromatin instability impairs the normal chromatin condensation, leading to a dysregulation of structural genes located in adjacent euchromatic segments.

With respect to immune response one candidate gene is the most recently discovered $T$ cell receptor gene, the zeta gene, which has previously been mapped to an area around the chromosome 1 centromere. ${ }^{30-32}$ Using fluorescence in situ hybridisation (FISH) in combination with the identification of gold particles by electron microscopy, we have recently mapped the zeta gene to chromosome band 1q23.1 (fig 2B), distal to the heterochromatic block in the long arm (Stacey M, Barlow A, Hultén $M$, unpublished data). We hypothesise that the normal functioning of the major heterochromatic blocks regulates expression of immune response genes, including the zeta gene. If this hypothesis is correct, we would then expect other immune response genes to be located adjacent to the heterochromatic blocks of chromosomes 9 and 16 . It seems likely that such regulation would be programmed during embryonic life, and thus the ICF syndrome may represent a maturation arrest in the programmed development of the immune response. It is of added interest that the same heterochromatic blocks are seen to be expanded during early normal embryological development, ${ }^{33}$ and also in the (immuno-incompetent) spermatocytes. ${ }^{3435}$ 
Heterochromatin undercondensation of the $\mathbf{Y}$ chromosome and spermatogenesis In 1969 it became apparent that quinacrine mustard specifically stains the heterochromatic block of the Y chromosome, identified by a bright fluorescence (L Zech, personal communication); and it was only later that it became clear that quinacrine staining in fact allows the identification of all the human chromosomes by virtue of a differential banding pattern along the length of each individual chromosome. ${ }^{15}$ The same banding pattern allows identification of male germ line autosomes at premeiotic and first meiotic metaphase. ${ }^{18}$

It was initially thought that the germ line differed from the soma in not showing a $Y$ heterochromatic block (Yqh) in premeiotic metaphases. ${ }^{36}$ However, more detailed studies showed quite clearly that the Yqh behaves differently in the different types of spermatogonia. Thus some spermatogonia with chromosomes of similar morphology to those of in vitro cultured lymphocytes had a normal appearing Y. Other spermatogonia, however, including two types with more compacted autosomes (presumably representing the later spermatogonial maturation stages $B$ and $C$ ), showed a striking undercondensation of the Yq, which sometimes could be seen expanded over the entire cell nucleus. ${ }^{345}$

With the introduction of FISH, using a probe specific for the $\mathrm{Y}$ heterochromatic block, it has been possible to confirm and extend these observations. Thus we have now shown that the Yqh is decondensed not only in some spermatogonial metaphases but also in interphase nuclei. ${ }^{37}$ Examples are seen in fig 3. We suggest that this differential Yq heterochromatin behaviour represents a programmed activation of adjacent spermatogenesis genes, ${ }^{38}$ and vice versa that the somatic compact Yq heterochromatin ensures the silencing of such genes at stages other than in the germ line. In conclusion we propose that also in this situation the condensation pattern of the heterochromatin block represents a system that allows modification and regulation of tissue and cell specific gene activation and transcription.

\section{Concluding remarks}

We have suggested that the compaction status of repeated DNA sequences may provide a mechanism for regulation of the tissue specific transcriptional machinery of structural genes, located in adjacent euchromatin in the human genome. This hypothesis, which constitutes a modification of a well known Drosophila model, the so called heterochromatin position effect variegation (PEV), is based on the heterochromatin instability that we have observed in two different situations in humans. Thus we have described firstly how the $T$ cell specific instability/decondensation of the main autosomal heterochromatic blocks in the ICF syndrome may represent a developmental maturation arrest resulting in dysregulation of $\mathrm{T}$ cell specific immune responses. Secondly, we propose that the undercondensation of the $Y$ heterochromatic block in certain types of spermatogonia provides a mechanism for the normal regulation of spermatogenesis genes, located in adjacent euchromatin.

The proposed mechanism for the switch on/ off processes of structural genes as formulated from these examples, and which we have called HIT, may well be common and, with further modifications, generally applicable. Thus, for example, it has recently become apparent that amplification of non-coding repeated DNA sequences per se may in a variety of situations influence the expression of adjacent structural genes. Examples of such situations include common diseases such as the fragile $\mathrm{X}$ mental retardation syndrome (FRAXA), myotonic dystrophy, insulin dependent diabetes mellitus, and certain cancers. ${ }^{24}{ }^{25-41}$ It should be added that somatic instability, which is a general characteristic feature of any expanded arrays of repeated DNA sequences, ${ }^{11}$ implies a repeat length "mosaicism" between cells and tissues. The overall phenotypic effect will accordingly depend on the relationships between this type of size variation, the relative position of the genes concerned, and the functional phenotypic effect of the relevant gene expressivity.

It seems reasonable to conclude that the so called junk DNA may indeed provide a versatile system which, by way of its unique and differential properties of condensation and amplification, may be looked upon as most valuable and treasured items that are specifically involved in the complex modulation of normal tissue and cellular gene activity. One particularly interesting aspect of our HIT and RUN hypothesis concerns the precise localisation of genes differentially switched on during development, where the progressive chromatin condensation status, as regulated by adjacent heterochromatin (fig 1), may play a role. No doubt, once attention has now focused on the potential functional role of repeated DNA sequences in the human genome, further knowledge will rapidly accumulate about the other side of this antique coin, that is, the controlling mechanisms and specific proteins involved in regulating reapeated DNA sequences and heterochromatin behaviour in itself. $^{21}$

This work is supported by the Letten F Saugstad Fund and the Leverhulme Trust.

1 Muller HJ. Types of visible variations induced by X-rays in Drosophila. F Genet 1930;22:299-334

2 Muller HJ. Further studies on the nature and causes of gene mutations. Proc Int Congr Genet 1932;1:213-25.

3 Henikoff S. Position-effect variegation after 60 years. Trends Genet 1990;6:422-6.

4 Shaffer CD, Wallrath LL, Elgin SCR. Regulating genes by packaging domains: bits of heterochromatin in euchromatin? Trends Genet 1993;9:35-7.

5 Verma RS, ed. Heterochromatin: molecular and structural aspects. Cambridge: Cambridge University Press, 1988.

Heitz E. Das Heterochromatin der Moose. I fahrb Wissensch Bot 1928;69:762-818.

7 Choo KH, Vissel B, Nagy A, Easly E, Kalitsis P. A survey of the genomic distribution of alpha satellite DNA on of the genomic distribution of alpha satellite DNA on
all the human chromosomes, and derivation of a new all the human chromosomes, and derivation of a new
consensus sequences. Nucleic Acids Res 1990;19:1179-82. 8 Kipling D. The telomere. Oxford: Oxford University Press, 1995.

9 Verma SM. Heteromorphisms of heterochromatin. In: Verma RS, ed. Heterochromatin: molecular and structural aspects. Cambridge: Cambridge University Press, 1988 276-92.

10 Lichter P, Cremer T. Chromosome analysis by non-isotopic in situ hybridization. In: Rooney DE, Czepulkowski BH, 
eds. Human cytogentics: a practical approach, vol I. Constitutional analysis. New York: JRL Press, 1992;157:92.

11 Vogt P. Potential genetic functions of tandem repeated DNA sequence blocks in the human genome are based on a highly conserved "chromatin folding code". Hum Genet 1990;84:301-36.

12 Armour JAL, Jeffreys AJ. Biology and applications of human minisatellite loci. Curr Opin Genet Dev 1992;2:850-6.

13 Miwa S. Triplet repeats strike again. Nature Genet 1994; 6:3-4.

14 Rattner JB, Lui CC. Radial loops and helical coils coexist in metaphase chromosomes. Cell 1985;42:291-6.

15 Caspersson T, Zech L, Johansson C, Modest EJ. Identification of human chromosomes by DNA-binding fluorescent agents. Chromosoma 1970;30:215-27.

16 Seabright $M$. A rapid banding technique for human chromosomes. Lancet 1971;ii:971-2.

17 Jhanwar SC, Burns JP, Alanso ML, Hew W, Chaganti RSK. Mid-pachytene chromosome maps of human autosomes. Cytogenet Cell Genet 1982;33:240-8.

18 Caspersson T, Hultén M, Lindsten J, Zech L. Identification of chromosome bivalents in human male meiosis by quinacrine mustard fluorescence analysis. Hereditas 1971;67:147.

19 Dorer DR and Henikoff S. Expansions of transgene repeats cause heterochromatin formation and gene silencing. Cell 1994; 77:993-1002.

20 Henikoff S, Loughney K, Dreesen TD. The enigma of dominant position-effect variegation in Drosophila. In: Heslop-Harrison JS and Flavell RB. The chromosome. Dorchester: Bios Scientific Publishers, 1994.

21 James TC, Elgin SCR. Identification of a non-histone chromosomal protein association with heterochromatin in Drosophila melanogaster and its gene. Mol Cell Biol 1986;6: 3862-72.

22 Prokofieva-Belgovskaya AA. Cytological properties of inert regions and their bearing on the mechanisms of mosaicism and chromosome rearrangement. Dros Int Serv 1941;15: 34-5.

23 Tartof KD, Henikoff S. Trans-sensing effects from Drosophila to humans. Cell 1991;65:201-3.

24 Green M, Krontiris TG. Allelic variation of reporter gene activation by the HRSA1 minisatellite. Genomics 1993;17: 429-34.

25 Krontiris TG, Devlin B, Karp DD, Roberts NJ, Risch N. An association between the risk of cancer and mutations in the HRAS1 minisatellite locus. $N$ Engl f Med 1993; 329:517-23.

26 Hulten M. Selective somatic pairing and fragility at $1 \mathrm{q} 12$ in a boy with common variable immunodeficiency. Clin Genet 1978;14:294.

27 Tiepolo L, Maraschio P, Gimelli G, Cuoco G, Gargani GF, Romano C. Multibranched chromosomes 1,9 , and 16 in a patient with combined IgA and IgE deficiency. Hum Genet 1979;51:127-37.
28 Smeets DFCM, Moog U, Weemaes CMR, Vaes-Peeters G, Merk GFM, Niehof JP, et al. ICF syndrome: a new case and review of the literature. Hum Genet 1994;94:240-6.

29 Stacey M, Bennett MS, Hultén M. FISH analysis on spontaneously arising micronuclei in the ICF syndrome. $\mathscr{f} \mathrm{Med}$ taneously arising micro

30 Weissman AM, Hou D, Orloff DG, Modi WS, Seuanel H, O'Brien SJ et al. Molecular cloning and chromosomal localisation of the human $T$ cell receptor zeta chain: distinction from the molecular CD3 complex. Proc Natl Acad Sci USA 1988;85:9709-9713.

31 Modi WS, Weissman AM, Seuanel H, Klausner RD, O'Brien SJ. Chromosomal localisation of the T-cell receptor zeta chain. Cytogenet Cell Genet 1989;51:1047.

32 Jensen JP, Hou D, Ramsburg M, Taylor A, Dean M, Weissman AM. Organisation of the human T cell receptor zeta/ $n$ gene and its genetic linkage to the FcgRii-FcgRiii gene $n$ gene and its genetic linkage to the Fcer
cluster. F Immunol 1992;148:2563-71.

33 Pérez MM, Miguez L, Fuster C, Miró R, Genesca A, Egozcue J. Heterochromatin decondensation in chromosomes from chorionic villus samples. Prenat Diagn 1991;11:697-704

34 Hultén M, Goldman ASH, Saadallah N, Wallace BMN, Creasy MR. Meiotic studies in man. In: Rooney DE, Czepulkowski BH, eds. Human cytogenetics: a practical approach, vol I, Constitutional analysis. New York: JRL Press, 1992:193-221.

35 Hultén M, Linsten J. Cytogenetic aspects of human male meiosis. In: Harris $\mathrm{H}$, Hirschhorn $\mathrm{K}$, eds. Advances in human genetics. New York: Plenum Press, 1973:327-87.

36 Pearson PL, Bobrow M. Fluorescent staining of the $Y$ chromosome in meiotic stages of the human male. $\mathcal{F}$ Reprod Fertil 1970;22:177.

37 Armstrong SJ, Kirkham AJ, Hultén MA. XY chromosome behaviour in the germ-line of the human male: a FISH analysis of spatial orientation, chromatin condensation and pairing. Chromosome Res 1994;2:445-52.

38 Kobayashi K, Mizuno K, Hida A, Komaki R, Tomita K, Matsushita I, et al. PCR analysis of the Y chromosome long arm in azoospermic patients: evidence for a second locus required for spermatogenesis. Hum Mol Genet 1994; 3:1965.

39 Hirst MC. Length variation in fragile X. In: Humphries SE, Malcolm S, eds. From genotype to phenotype. Dorchester: Malcolm S, eds. From genotype to pheno

40 Winchester CL, Johnson KJ. How a dynamic mutation manifests in myotonic dystrophy. In: Humphries SE, Malcolm S, eds. From genotype to phenotype. Dorchester: Bios Scientific Publishers, 1994;143-61.

41 Bennett ST, Lucassen AM, Gough SCL, Powell EE, Undlien DE, Pritchard LE, et al. Susceptibility to human type 1 diabetes at IDDM2 is determined by tandem repeat variation at the insulin gene minisatellite locus. Nature Genet 1995;9:284-92. 